\title{
HISTORIA DEL DESARROLLO EMPRESARIAL COLOMBIANO
}

Jesús Alberto Ramírez*

* Profesor Departamento de Administración Universidad de Pamplona. 


\section{HISTORIA DEL DESARROLLO EMPRESARIAL COLOMBIANO}

\section{RESUMEN}

El presente escrito hace parte de un trabajo que se acerca a una explicación de la historia del desarrollo empresarial colombiano y su escasa competitividad en el mercado nacional e internacional, derivada de la baja productividad como consecuencia de factores estructurales internos y de la dependencia respecto a los modelos de desarrollo económicos y administrativos implementados desde afuera, modelos que interpretan la realidad de países industrializados a hoy llamados postmodernistas, pero que no encajan en las sociedades tercermundistas, si se quiere subpostmodernizadas, porque no responden a la realidad concreta y especifica de Latinoamérica y en particular de Colombia.

Palabras Claves: Desarrollo, Historia, Empresarial, Competitividad, Mercado, Realidad, Países, Modelos Económicos, Modelos Administrativos, Burguesía. Dependencia, Estados Unidos, Latinoamérica. Colombia.

\begin{abstract}
This document is part of a work that is closer to an explanation of the history of Colombian business development and low competitiveness in the domestic and international, from the low productivity due to internal structural factors and the dependence on the economic development models and administrative implemented from outside models to interpret reality in industrialized countries today called post-modernists, but that do not fit in the third world societies, if you will subpostmodernizadas, because they respond to the specific factual and Latin America and Colombia in particular.
\end{abstract}

Key Words: Development, history, business, competitive, market, Reality, Countries, Economic Models, Models Administration, Bourgeoisie. Unit, U.S., Latin America. Colombia. 


\section{ANTECEDENTES $1850-1930$}

La estructura económica y social que se ha conformado en Colombia ha sido pasiva, debido a fuerzas que operan primordial mente desde el exterior, ha sufrido sus cambios sociales mas bien que promoverlos, se ha visto empujada a sus transformaciones estructurales por grandes cambios en la correlación de las fuerzas imperialistas, cambios en los que es natural ha tenido poco que ver.

La introducción del modelo del laissez - faire en Colombia a partir de 1850 obedece a los intereses ingleses que habían desarrollado las manufacturas por medio de su revolución industrial estableciendo la libertad de comercio, momento en la cual nuestro país se dedicaba al cultivo agrícola para exportarlo al mercado internacional y a la vez importaba manufacturas, brindando así la oportunidad a los terratenientes y grandes comerciantes criollos de desempeñar un papel más orgánico en el comercio exterior. De esta manera el viraje de nuestro país y en conjunto de América Latina abre una nueva fase hacia formas de dependencia colonial ${ }^{1}$, el llamado modelo de crecimiento hacia fuera. El cultivo de café que fue finalmente el producto agrícola que estableció Colombia como producto de exportación, después de haber hecho experimentos con el tabaco, el añil y la quina, creo el desarrollo y la formación del mercado interno, se obtuvo una mayor capacidad de consumo, hecho que fue posible debido a las condiciones sociales de su producción. ${ }^{2}$

A la vez éste condiciono el surgimiento de las industrias urbanas en Colombia con el objetivo de atender la demanda creciente de las regiones cafeteras.

Con el crecimiento y las fluctuaciones del comercio exterior la inestabilidad y la dependencia de la economía local aumentaban proporcionalmente y se generaban graves crisis domesticas así como también en algunos casos procesos significativos de crecimiento. $^{3}$

En las épocas que preceden al auge y predominio de la producción del café, la cultura Colombiana es una cultura humanista y abstracta. ${ }^{4}$ Existía una reposada vida económica, se cultiva y se produce lo necesario para el consumo familiar. Hay costumbres coloniales, sincera devoción religiosa, hay temor a los cambios y a las transformaciones por leves que estas sean. La expansión y el auge del cultivo del café modifican totalmente esa situación. La economía nacional es insertada en la economía mundial, desaparece la producción para el consumo inmediato familiar, de un mercado local y se incrementa el cultivo del café hacia el mercado externo.

1 Arrubla Mario, "Estudios Sobre el subdesarrollo Colombiano". Editorial la Carreta.1984.

2 Nieto Arteta L, "El café en la sociedad colombiana”, Editorial Ancora, 1985.

${ }^{3}$ Mc Greevy, Paul "Historia Económica de Colombia 1845 - 1930" Editorial Tercer Mundo V edición 1985.

${ }^{4}$ Nieto Arteta L, Ibíd. 
La moderna industria en Colombia se desarrollo y configuro sus bases a comienzos del pasado siglo, grandes empresas de textiles, bebidas, tabaco, fósforos, dulces y galletas, cementos y vidrios. Ya en 1916 operaban fábricas como Coltejer, Postobon, Noel, Coltabaco, la Garantía y cementos Samper empresas que a hoy mantienen su nivel de importancia. Mención especial merece el caso de la industria cervecera que trae consigo la aculturación de un producto nuevo para el país y que inicia su proceso empresarial años atrás. Se considera 1889 la fecha cuando se da inicio a la fabricación moderna de la cerveza en Colombia; producto que va atender un mercado de la clase media y alta especialmente en la ciudad de Bogotá, la verdad es que la historia cervecera en Colombia inicia muchos años antes, con la producción artesanal de la cerveza en diferentes sitios del país, pero el empresario más recordado es don Leo kopp ciudadano alemán radicado en Colombia, en parte por su empuje y en parte porque Bavaria (Sab Miller), es a hoy la empresa mas antigua y prestigiosa del país.

Las divisas obtenidas del comercio exterior del café posibilitaron la compra de bienes de capital y con ello, la conformación de una base industrial. El mercado local se impregna de una circulación monetaria y acumulación de capital posibilitando el crecimiento del mercado interno. Además, éste mismo fenómeno quiebra parcialmente las relaciones de tipo precapitalista y modifica en alguna forma la renta del suelo.

Este proceso de industrialización no fue ajeno al replanteamiento de la política económica efectuada en 1880, que resolvió finalmente el largo debate sobre protección y libre cambio que llevaba más de medio siglo y se establecieron políticas proteccionistas a la industria nacional mediante fuertes regímenes aduaneros, iniciando con la ley de 1880 y que se mantuvo durante toda esa época.

Hay que anotar, que pese a estos cambios, la economía colombiana hasta 1920 era estructuralmente agraria, con bajos niveles de productividad y con nulos niveles de incorporación tecnológica. ${ }^{5}$ En 1925 la industria solo representaba el 10\% del producto nacional. También se registra un flujo migratorio hacia las poblaciones urbanas debido al incipiente desarrollo industrial y la creciente construcción de obras públicas, que reflejaron una diferenciación de los salarios entre actividades agrícolas y no agrícolas.

Desde el año de 1918 se iniciaron las primeras luchas y organizaciones beligerantes de la clase obrera, enfrentando dura represión oficial, culminando este periodo con la sangrienta matanza de las bananeras, donde el principal patrón era el imperialismo Norteamericano y el propio Estado colombiano.

Al finalizar este periodo, el poder económico de los Estados Unidos lo confirma como el nuevo centro hegemónico de poder. Este auge económico tiene como efecto inmediato

\footnotetext{
${ }^{5}$ Afirmación hecha por el maestro Jesús Antonio Bejarano, en historia Económica de Colombia, Compilador Jesús Antonio Ocampo, Editorial Tercer Mundo, Bogotá 1994
} 
aumentar la demanda de materias primas y productos agrícolas, quedando Colombia insertada bajo la influencia del imperialismo norteamericano.

\section{DESARROLLO DE LA INDUSTRIA DESPUES DE LA CRISIS (1929)}

La crisis mundial del capitalismo tiene centro en los Estados Unidos ,presentándose a finales de 1929 y sus efectos se propagan a la mayoría de las potencias de ese entonces afectando lógicamente de forma considerable a los países dominados, dadas las relaciones orgánicas con las potencias económicas. La depresión tiene efecto inmediato, la caída de los precios, originada en la superproducción de mercancías durante los años anteriores a la crisis, periodo en el cual se expande al aparato productivo norteamericano de una forma excepcional, marcando su punto más alto durante el primer cuatrienio de este siglo.

El momento de iniciación de dicha crisis lo marca la bancarrota de la bolsa de valores de Nueva York en el otoño de 1929, consecuencia de la quiebra y cierre de las empresas y a partir de este momento se origina una situación de paro forzoso en la economía de los Estados Unidos que tiene como efecto un descenso en el consumo nacional hasta el $40 \%$ del nivel corriente.

El cierre de los mercados extranjeros conjuntamente con el descenso en la capacidad para importar disminuye drásticamente la compra de productos manufacturados en el exterior, lo cual brinda coyuntura para dar un impulso a las industrias colombianas, que se formaron en los años anteriores a la depresión, por medio de la sustitución de importaciones. Esto incide mas en el aumento de la producción de artículos de consumo, con cierto grado de elaboración que en el número de nuevas fabricas instaladas.

En lo social se acentuaron las movilizaciones campesinas y obreras que había comenzando a manifestarte en el país antes de la crisis y se hicieron importantes avances en reforma agraria y laboral. En el plano político el país vivió los primeros gobiernos laborales, en la esfera económica se experimenta una marcada transición en el modelo de desarrollo, surgiendo como resultado muchos instrumentos básicos de intervención del estado (control de cambios, régimen fiduciarios organizados alrededor del banco de la república, impuesto moderno de renta y patrimonio entre otros). El país experimenta un rápido ritmo de crecimiento industrial inigualado en el resto de América latina y en su historia posterior.

El proceso de recuperación de la industria, posterior a los años de crisis, puede apreciarse en el crecimiento de las cifras de la importación de maquinaria y en cifras de la producción industrial. 
En esta época surgen las sociedades anónimas para la industria fabril, gracias a estas se pudo acopiar los cuantiosos recursos financieros requeridos para industrializar al país, sistema que popularizó la inversión industrial y asimilo el ahorro, atrayendo hacia la industria multitud de pequeñas sumas.

Particularmente fueron notables los aumentos de la producción de cerveza, gasolina, electricidad industrial y cemento.

Entre 1930 y 1993 se fundaron 842 establecimientos industriales, la mayor parte en renglones fabriles, que antes no existían. Esto significo la incorporación a nuestra economía de numerosas tecnologías nuevas y de conocimientos en otras tantas actividades industriales tales como: Preparación de conservas vegetales, elaboración de derivados del maíz, trabajo de metales, vulcanizados del caucho, malterías, etc.

Con la llegada al gobierno de Alfonso López Pumarejo para el año 1934 concluye lo que pudiera llamarse la fase de industrialización espontanea, durante la cual algunos empresarios colombianos, con recursos nacionales lograron crear una base fabril para el país, constituida por empresas de las que solo un pequeño número empezaba a adquirir características de fabricas modernas. En esa etapa se comenzó (A fines del segundo decenio), a construir realmente el inicio de la industrialización y la firmeza de su base económica, queda confirmada por el hecho de que ni aún la gran crisis logro frustrar su desarrollo.

A partir de 1935, con nueva filosofía política, el gobierno asume un decisivo papel en el manejo de la economía, en respaldo del desarrollo industrial. La orientación de este gobierno se concreto con varios estatutos que se expidieron en 1936. El mas importante fue la reforma constitucional, que consagro la intervención del estado en la economía. Se expidió también una reforma tributaria que creo en el país los instrumentos de la tributación directa y universal, la tarifa progresiva, el tratamiento diferencial a tipos y tamaños de empresas, las deducciones por depreciación y muchos otros elementos del régimen de impuestos que habrían de incidir fundamentalmente en la industrialización. La ley de tierras (ley 200) se expidió también en ese año. Con ella se aspiraba a iniciar en el país una urgente reforma agraria, que hubiera podido tener impacto benéfico como estimulo de la industrialización a través de la ampliación de los mercados, pero, que no pudo cumplir su cometido por que fue desvirtuada por sus opositores.

En todo caso en el periodo de 1936 a 1939, se iniciaron nuevos renglones industriales importantes: paños de lana, acero de varilla, gases industriales, grasas industriales y otros. Además se ampliaron y modernizaron renglones como los de cervecería, envases de vidrio y textil de algodón. 
Puede llegar a afirmarse paradójicamente que de algún modo la crisis capitalista en el ámbito mundial, sirvió para que el país creara una base firme para el desarrollo industrial capitalista que requería y que se ha mantenido a lo largo de toda su historia.

\section{CONSOLIDACION DEL CAPITALISMO MODERNO EN COLOMBIA}

\section{Crecimiento y cambio estructural: \\ (Tendencias del desarrollo colombiano en la segunda postguerra 1945-1980).}

Durante este lapso, el producto interno bruto (PIB) de Colombia se multiplico por siete, aunado a cambios en el crecimiento demográfico el cual fue bastante acelerado que junto con el descenso de la mortalidad y mejoramiento en el nivel de calidad de vida propicio un alto crecimiento. Este hizo parte de la bonanza más espectacular que haya experimentado la economía mundial en su historia. Sin embargo, el crecimiento del producto por habitante en Colombia fue similar al del resto de América latina. En consecuencia, la distancia que separaba a Colombia y a los países en desarrollo de las economías industrializadas lejos de estrecharse se amplió.

El crecimiento económico se vio acompañado de un cambio estructural en la composición de la actividad económica, en la medida que se hizo más notoria la reducción de la participación del sector agropecuario en la economía. La disminución en el tamaño relativo del sector agropecuario dio paso al surgimiento y consolidación de nuevas actividades económicas, en especial la industria manufacturera, aunque también en los sectores de transportes, financiero, comunicaciones y servicios públicos modernos.

El avance relativo del sector manufacturero fue particularmente rápido en las décadas del cuarenta y cincuenta, continuando el impulso que se había iniciado en los treinta. Su avance se desacelero a mediados de los años sesenta y setenta; a fines de este último empezó a presentar un importante retroceso, preludio de la crisis de los años ochenta.

Tal proceso de desarrollo permitió la acumulación de capital privado y social más importante en la historia del país. En el frente privado los aspectos mas sobresalientes fueron la construcción de grandes fabricadas modernas y empresas agro-industriales; en el aspecto social lo mas significativo fue la formación de capital humano.

La recomposición de la actividad económica del país y la acumulación de capital origino una alta movilidad social, en especial las de la actividad agropecuaria hacia las fronteras agrícolas y la redistribución demográfica. 
Los cambios en la composición de la actividad económica se reflejaron también en la estructura de empleo, donde pierde importancia relativa el sector primario, lo que origino así mismo la consolidación del trabajo asalariado, típico del capitalismo moderno.

La transformación de la economía tuvo también un impacto notorio sobre la estructura regional del país. Hasta mediados del siglo los grandes beneficiarios del proceso de desarrollo habían sido las cuatro ciudades mas industrializadas del país y la zona cafetera. A partir de 1950 el patrón de desarrollo regional sufrió modificaciones importantes. Bogotá continuo concentrando una proporción creciente de la actividad económica y de la población; por el contrario, dos de los departamentos más industrializados, Antioquía y Atlántico, se expandieron a ritmos inferiores al promedio nacional; los departamentos cafeteros entraron en franca declinación. Esta tendencia marcada es la continua concentración de la actividad económica en Bogotá y además las oportunidades generadas por el proceso de desarrollo que beneficia a regiones que hasta 1950 eran ajenas al desarrollo de la actividad económica.

En las cuatro décadas posteriores a la segunda guerra mundial el desarrollo empresarial COLOMBIANO ha tenido dos características sobresalientes, la primera de estas fue el resultado del proceso de industrialización que había vivido el país durante los años treinta y hasta la segunda guerra mundial, la segunda característica ha sido el lento dinamismo de las exportaciones. La explicación de esta tendencia de las exportaciones debe buscarse en el comportamiento de las ventas externas del café, pero principalmente en la diversificación excesivamente lenta de las bases exportadoras.

Con las exigencias propias de la segunda guerra mundial aparecen las primeras formas específicas de la intervención estatal, los primeros avances de la inversión extranjera (USA) en la industria, las primeras producciones de bienes intermedios, etc. De especial importancia fueron la fundación del IFI y el establecimiento del control de precios por parte del gobierno, modernizando la contabilización y el control del gasto publico.

En esos años avanzaron en su empleo y en su conocimiento la tecnología del frio (especialmente en la industria cervecera y de alimentos); la sustitución de antiguos telares mecánicos en la industria textil, por los nuevos telares automáticos y el montaje de rotativas tipográficas de alta velocidad en las imprentas de los periódicos, en remplazo de las antiguas prensas mecánicas. Síntoma significativo del desarrollo industrial durante la segunda guerra, es el consumo de electricidad industrial.

La reforma constitucional de 1968, ratificó la vigencia de la libertad de empresa, pero a su vez amplio las facultades de intervención del Estado. Estableció, de una parte, que la dirección general de la economía estaría a cargo del Estado, quien llevaría a cabo tal función orientadora por intermedio de la planificación, con miras a lograr pleno empleo de los recursos, el mejoramiento social (especialmente los grupos menos favorecidos) y el 
desarrollo integral. De otra parte, la reforma concentro un mayor poder decisorio en manos del poder ejecutivo a fin de agilizar el manejo de los instrumentos de intervención. Ello se tradujo en una mayor injerencia del presidente de la república en el manejo monetario, en las políticas de ahorro, en el crédito publico, en la organización y reforma de los aspectos relativos al comercio exterior y la administración de los institutos descentralizados. Así mismo, centralizo en el poder ejecutivo la iniciativa legislativa en materia de gasto público y restringió la iniciativa parlamentaria en lo relativo al régimen tributario.

Por otro lado, a nivel del desarrollo empresarial esta época (1929-1980), se caracterizo por la asimilación de practicas Tayloristas, fomentadas por la escuela de minas de Medellín, la universidad Nacional e Incca de Bogotá (Cada una con una aplicación diferencial de la teoría). Este tipo de organización generó un cambio en las relaciones obrero-patronales en las que prevaleció el capital sobre el trabajo y se empezaron a manejar conceptos como el de productividad y eficiencia.

Así mismo, se observa que la influencia de los modelos económicos extranjeros, se reflejo en el modelo económico al interior del país y el tipo de modelo de desarrollo empresarial asumido. Ahora bien, dicha influencia se manifestó en la empresa básicamente en la organización interna y en la búsqueda de productividad, pero no se manifestó de igual manera para el concepto de comercialización externa, debido a que el mercado era básicamente de carácter local, lo cual genero que la empresa colombiana no manejara el concepto de competitividad con relación al mercado interno y externo. En varias empresas se mantuvo un tipo de "gestión empresarial de carácter especulativo orientado fundamentalmente hacia la negociación con entidades gubernamentales y financieras de las que dependían las decisiones que determinaban la rentabilidad; en mayor grado que la innovación tecnológica y la modernización empresaria". ${ }^{6}$

Paralelo a lo interior, aparecen el modelo FORDISTA mas conocido como "modelo de producción en masa" este se convirtió en un paradigma de las políticas económicas y sociales de las mayorías de las regiones del mundo; América latina, bajo la influencia del CEPAL y de acuerdo con los postulados sobre "desarrollo hacia dentro" o sustitución de importaciones, genero en la región un proceso de industrialización con dos importantes limitaciones: las tecnologías fragmentarias y los mercados pequeños (fajnzylber 1983).

En Colombia las plantas de producción eran pequeñas y no podían competir a nivel internacional, lo que llevo como compensación, el establecer una amplia estructura de proteccionismo (arancel altos, subsidios, etc.), a fin de mantener cautivos los mercados internos.

\footnotetext{
${ }^{6}$ Afirmación hecha por la doctora Anita Weiss en su texto la empresa colombiana entre la tecnocracia y la participación, Editorial Universidad nacional, 1990
} 
Los sistemas industriales latinoamericanos desarrollaron una escasa capacidad de especialización y exportación, dándose la paradoja de que la. Importación de partes e insumos necesarios para la producción, dependían de la capacidad de generación de divisas de las exportaciones del sector primario; la precaria distribución de ingresos y la alta tasa poblacional conllevan a la creación de sistemas industriales fácilmente monopolizables y poco dinámicos, que no generan suficiente empleos y tienen alto porcentaje de ociosidad (Rojas 1980).

La amplia participación del estado, que a su vez era regulador y empresario le permite establecer con el mercado interno estrechas relaciones de dependencia y clientelismo dando origen a una burguesía o clase empresarial eminentemente "rentista".

En Colombia, las teorías clásicas de administración, introducidas desde las primeras décadas del siglo XX, tiene su mayor auge de, aplicación en la década de los cincuenta cuando se produce su implementación masiva en diversas empresas del país, a través de la práctica de la ingeniera industrial orientada a racionalizar la producción del trabajo. Para ello se contratan compañías asesoras norteamericanas y se forman ingenieros en el exterior; sólo a finales de esta década se organizaron las primeras facultades de ingeniería industrial del país. ${ }^{7}$

El estudio realizado por SAVAGE en 1960, tres empresas de Medellín, muestra como la introducción del taylorismo permitió el remplazo de las relaciones tradicionales propias del régimen patronal a un nuevo régimen de los "doctores", en el cual no solo se limitaba a la organización del trabajo, sino que se convirtió en el "Centro Emocional de su Mundo".

Entre las empresas que se vieron influenciadas por este modelo tenemos: Corona, Evertif, Fabricato, Icollantas entre otras.

La crisis del petróleo de 1974, fue el detonante que acelero el quiebre del fordismo (tironi y lagos, 1991). Los factores condicionantes de la crisis fueron por una parte la saturación de los mercados y la mayor volatilidad de la demanda, que se manifestaba en la limitación de crecimiento de muchos sectores y productos, y en ciclos de vida más cortos en el caso de otros, por lo tanto las grandes instalaciones e infraestructura dejan de ser funcionales, ya que no permiten las economías de escala necesarias para compensar los altos costos fijos generados en la producción. Se produce un creciente agotamiento de la organización fordista en la lucha por la productividad y las innovaciones increméntales.

\footnotetext{
${ }^{7}$ Años atrás se habían realizado intentos en la Universidad Nacional 1939 y en 1920 la Escuela de minas de Medellín ya aplicaba ciertas técnicas solo hasta 1958 se funda la primera facultad de ingeniería industrial en la Universidad Industrial de Santander.
} 
Las crisis de América Latina tomaron diferentes formas; por ejemplo, en Brasil y en el cono sur se caracterizo por la necesidad de expansión de la industria regional "etapa difícil" de sustitución de medios intermedios y de capital, así como de ampliación de los mercados y la aceleración de los procesos de integración social.

\section{NUEVAS CARACTERISTICAS DEL DESARROLLO INDUSTRIAL COLOMBIANO $(1980 \ldots)$}

Las repetidas recesiones económicas de los setentas y ochentas han provocado una larga fase de acumulación muy lenta, caracterizada por cambios tecnológicos profundos, creando un gran numero de desempleos y la restructuración industrial a lo largo y ancho del mundo capitalista.

Durante este periodo la industria colombiana se encuentra en una etapa de crecimiento recesivo, de crisis para muchas empresas que buscan adecuarse a las nuevas condiciones del mercado mundial, el cual ya no se orienta por una producción masiva y estandarizada tendiente a disminuir costos sino que se trata de un mercado cambiante con tendencias a la diferenciación, con demandas especificas y variadas, en un proceso de crecimiento intensivo donde la principal fuerza productiva es la ciencia y sus aplicaciones tecnológicas. $\mathrm{Al}$ igual que otros países de América latina, Colombia toma medidas para controlar la dependencia de su industria frente al sector publico, privatizando una serie de entidades estatales y "flexibilizando" la contratación de la mano de obra a través de sucesivas reformas laborales, el diseño de una política anti-inflacionaria, al igual que el anuncio de políticas de modernización en áreas como el transporte, educación, salud, ciencia y tecnología. Sobre estas nuevas bases se esperaba que Colombia lograra una nueva fase de reconversión industrial, crecimiento y desarrollo, lo cual se ha logrado parcialmente debido a las situaciones coyunturales de orden político y económico, medidas que fueron impuestas por la banca multilateral a través del fondo monetario internacional, con el objetivo de facilitar y asegurar rentabilidad a la inversión extranjera.

La globalización de la economía con su libre flujo de capitales entre países define donde localizar las inversiones según le ofrezca mejores condiciones y de donde pueda exportar libremente a cualquier país del mundo. Esta es la verdadera fuerza material del libre cambio.

Además, estos cambios en el mercado generaron variaciones en la formas de consumir, que alteraron los hábitos ciudadanos. Mientras el ciudadano de ayer estuvo asociado a la capacidad de apropiarse de los bienes y formas de usarlos, el ciudadano de hoy, según afirmación de Néstor García, "se identifica en mayor medida con el consumo masivo de bienes que no representan las necesidades fundamentales de la sociedad y omitiendo su participación en el espacio publico y en el libre juego de la democracia”. 
Así, la economía norteamericana disfruto de una expansión en cierta forma patológica que se extendió hasta 1988 y que luego indujo una etapa de recesión. América latina y Colombia se vieron forzadas a asumir una deuda externa cuyo monto variaba de acuerdo con la política monetaria norteamericana, causando en la economía nacional la típica "crisis de la deuda externa" de la década de los 80.

En el escenario internacional, el derrumbe del muro de Berlín en 1989, la posterior caída de la unión soviética y la instauración del capitalismo centralizado en china conllevan a un nuevo orden económico mundial, en el cual las democracias parlamentarias desarrollan economías mas gobernadas por el mercado que por la intervención estatal. La nueva situación hace perder el sentido de izquierda y derecha; en los países del Este europeo la izquierda desapareció como tal y quedaron conservadores y liberales (Que revivieron después de cincuenta años de decadencia) y centrista. En los países capitalistas la división es entre intervencionistas, proteccionistas y neo liberales y surgen así los grupos ecologistas.

América latina y Colombia comenzaron a salir de la década perdida después de penosos ajustes macroeconómicos y dejar de poseer una multitud de empresas publicas que contribuían al déficit fiscal y prestaban pésimos servicios a la población; también tuvo que alcanzar grandes excedentes en servicio de su deuda externa que solo se vio aliviada en la medida en que el mercado financiero internacional regresó a tipos de interés moderados. En esta etapa Colombia después de haber llegado a promedios arancelarios del 45 y $80 \%$ los redujo a tasas del $10 \%$, permitiendo reasignación de importantes mercados en distintos países, abriendo una senda dinámica de inversiones mutuas dentro del marco liberal y de apertura económica, legalizando economías subterráneas con la libertad de cambios e importaciones, despenalización de las operaciones como el movimiento de capitales y contrabando, que en la practica surgieron antes sin cortapisa alguna.

La empresa colombiana, para dar respuesta a los nuevos retos de la economía internacional, adopta parcialmente en algunos sectores principios productivos y administrativos del modelo japonés, comúnmente llamado "Toyotista". Tal modelo esta conformado por un sistema cuya implantación remite originalmente a factores históricos y culturales propios de la sociedad japonesa. Si hubiese que resumir en tres palabras los ingredientes del éxito y el resultado esencial de la experiencia económica japonesa seria: tecnología, productividad y competitividad. Allí está la fuente y fruto principal de lo que este modelo económico produce al combinarlo de diferentes maneras, lo que otros o no pueden o se tardan mas en producir a pesar de usar los mismos ingredientes.

Por otra parte, la planeación estratégica, el servicio al cliente interno y externo, el mejoramiento continuo, los programas justo a tiempo, cero inventarios y cero errores, los 
círculos de calidad y de participación incluidos en la designación global como programas de calidad total, contribuyeron básicamente al despegue de la nueva era industrial. En Colombia, los procesos de cambio que se pueden ubicar en la dependencia, orientada al establecimiento de la organización como un sistema integrado con elementos de gestión participativa, tienen solamente una aplicación parcial y no han logrado desplazar a las relaciones tayloristas y clásicas prexistentes; lo relevante es resaltar el sentido cualitativa mente diferente de estos procesos dentro de nuevos parámetros. En algunas empresas se introducen programas de calidad total combinándolos con procesos de automatización de la producción, en otras implica reformas simplemente administrativas. Estos procesos de cambio parcial tal vez nunca conducirán a un cambio global en estas empresas, en otras, se viene desarrollando un proceso de cambio paulatino cuyo alcance solo se podrá evaluar a mediano o largo plazo.

El mercado mundial ya no tiende hacia la producción masiva y estandarizada orientada a disminuir costo, ahora se trata de un mercado cambiante con tendencias hacia la diferenciación, con demandas específicas y variadas (Nichos), en un proceso de crecimiento intensivo, en el cual la ciencia y la tecnología constituyen la principal fuerza productiva. Esto requiere mayor responsabilidad por parte de todos los que trabajan en las empresas y su vinculación activa para responder en forma rápida y eficiente a la diferenciación de los productos, mayor calidad de los mismos, mejoramiento de los procesos, innovación y la resolución de los problemas en general. Se responde a estos cambios por medio de automatización de la producción y la sistematización de la administración, buscando una integración y coordinación entre la diferentes áreas, lo que implican la creación de una organización concebida como un sistema integrado, en la cual se busca fomentar la participación e integración de sus empleados y trabajadores, con un nivel mayor calificación y responsabilidad para responder a las exigencias del incremento de la productividad, la diversificación de la producción y la calidad.

Al llegar a éste tercer milenio, podemos afirmar que el país aun se encuentra muy lejos de lograr sus objetivos a pesar del largo tramo de desarrollo capitalista que ha recorrido. Encontramos un país dividido entre los que tienen todo y los que no tienen nada, entre campesinos y terratenientes, entre patronos y trabajadores, entre ejército, paramilitares y guerrilleros entre estado y narcoterrorismo, entre participación y narcoparaestado; así se enfrenta a unas condiciones de lento crecimiento una veces y otras de franco estancamiento llevando a su población a una situación de profundo deterioro en su calidad de vida.

La ansiedad, es la marca de la sociedad colombiana que no se encuentra, ni sabe como canalizar sus energías sin explotar. Hay una crisis cultural en las nuevas generaciones abocadas a un futuro incierto, que parece no tener salida, especialmente con la desilusión que dejo la política radical y la muerte del socialismo. Los partidos tradicionales se han visto enfrentados a cambios muy rápidos y contundentes, propiciados por la inestabilidad 
creada por el narcotráfico y el paramilitarismo, que ha obligado a enfrentar la crisis con reformas y ampliación de los escenarios democráticos. El crecimiento económico se ha hecho mas lento en los últimos años, ha dependido demasiado de la economía del narcotráfico y es muy difícil recuperar los promedios de crecimiento logrados en el ultimo medio siglo, el desarrollo industrial ha perdido su ritmo y a pesar de las reformas neoliberales que buscan una nueva senda del crecimiento, no es claro que lo estén logrando pues la apertura de los ochenta condujo a un proceso revaluacionista que freno las exportaciones y el consecuente desarrollo económico y empresarial nacional.

Colombia es un país que cuenta con una dotación de recursos naturales, gente trabajadora y localización geográfica estratégica, que le permite estar bien posicionado para competir en el siglo veintiuno. Con solo muy pocos años de iniciado el proceso de apertura, es cada vez más claro su panorama económico y su futuro, a pesar que aun existen problemas fundamentales en el medio competitivo colombiano que deben ser resueltos de manera decidida y oportuna. Es necesario diversificar aun más su base exportadora, desarrollar el recurso humano, superar sus deficiencias de infraestructura, y mejorar sus condiciones internas para atraer la inversión extranjera.

Pero quizás lo más importante será el desarrollo de una actitud más agresiva hacia el aprendizaje y la modernización institucional como mecanismo de defensa frente al desarrollo mundial. Los modelos administrativos adoptados en el pasado brindaron un relativo éxito en su época que no corresponde a las necesidades de hoy, ni tampoco cumple con las exigencias de las organizaciones futuras, donde se requiere una estructura organizativa de gestión participativa, orientada a formar un sistema integrado basado en el principio cibernético según el cual la empresa se concibe y se maneja como un todo, donde cada una de las partes contribuyen al funcionamiento del conjunto a través de retroalimentación, para obtener una información que sirva de base para un proceso colectivo de toma de decisiones mas descentralizado.

Indudablemente el capitalismo trajo, a pesar de si mismo, cierto progreso económico y social, las luchas sociales lograron conquistas importantes en el campo social, político y cultural. Se venia avanzando en el respeto a la diferencia y la pluralidad económica, se han desarrollado nuevos modelos de participación ciudadana y de integración del estado, la comunidad y la clase empresarial ha buscando obtener los principios de subsidiariedad, cofinanciación y concurrencia plasmados en la carta magna. Pero esto no es suficiente, se debe seguir luchando por mejorar en todos los ambientes, en lo político, en lo económico, en lo social, en lo cultural, en la aplicación de la justicia, en lo tecnológico, científico y educativo, etc. para lograr tener el país que nosotros y nuestras descendencias se merecen. 


\section{BIBLIOGRAFÍA}

- Arrubla, Mario. “Estudios Sobre el subdesarrollo Colombiano”. Editorial la Carreta.1984.

- Bejarano, Jesús Antonio en historia Económica de Colombia, Compilador Jesús Antonio Ocampo, Editorial Tercer Mundo, Bogotá 1994.

- Fajnzylber, Fernando. La Industrialización Trunca de América Latina. México D.F. Editorial Nueva Imagen. 1983.

- García Canclini, Néstor: Consumidores y ciudadanos. Conflictos multiculturales de la globalización. Grijalbo. México. 1995.

- Ley 200 de 1936. Reforma Agraria.

- Martínez F, Carlos E. Administración de Organizaciones. Productividad y Eficiencia. Editorial Carlos Martínez. Imprenta Universidad Nacional. 1990.

- Mac Greevy, Paúl "Historia Económica de Colombia 1845 - 1930" Editorial Tercer Mundo V edición 1985.

- Nieto Arteta L. "El café en la sociedad colombiana", Editorial Ancora, 1985.

- Ortega Cárdenas, Alfonso. García Angarita, Héctor. Economía Colombiana .Ediciones Ecoe Segunda Edición.

- $\quad$ Rojas, Carlos Julio. Empresas Colombianas Exitosas. Editorial Grijalbo 1992.

- Tirado Mejía, Álvaro. Introducción a la Historia Económica de Colombia, Editorial la Carreta. Colombia 1984.

- Weiss de Belarcazar, Anita. La empresa colombiana entre la tecnocracia y la participación, Editorial Universidad Nacional, 1990.

- www.historiadelacocina.com 